\title{
Screening for intracranial aneurysms? Prevalence of unruptured intracranial aneurysms in Hong Kong Chinese
}

\author{
David Y. C. Chan, MRCS, ${ }^{1}$ Jill M. Abrigo, FRCR, ${ }^{2}$ Tom C. Y. Cheung, FRCR, ${ }^{2}$ \\ Deyond Y. W. Siu, FRCR, ${ }^{3}$ Wai S. Poon, FRCS, ${ }^{1}$ Anil T. Ahuja, MD, ${ }^{2}$ and George K. C. Wong, MD1 \\ 1Division of Neurosurgery, Department of Surgery, and 2Department of Imaging and Interventional Radiology, Prince of Wales \\ Hospital, The Chinese University of Hong Kong; and ' ${ }^{D}$ epartment of Diagnostic Radiology, Kwong Wah Hospital, Hong Kong, \\ People's Republic of China
}

\begin{abstract}
OBJECTIVE The objective of this study was to generate data on the local prevalence of unruptured intracranial aneurysms (UIAs) in asymptomatic Hong Kong Chinese individuals. First-degree relatives of patients with aneurysmal subarachnoid hemorrhage (aSAH) were recruited as surrogates of the general population and to explore the potential role of screening in this locality.

METHODS The authors identified first-degree relatives of consecutive patients with subarachnoid hemorrhage from a ruptured aneurysm who were admitted to a university hospital in Hong Kong from June 2008 to December 2010. Magnetic resonance angiography (MRA) was the imaging modality used to screen the cerebral vasculature of these asymptomatic individuals. If MRA showed abnormal findings, CT angiography was performed to confirm the MRA findings.
\end{abstract}

RESULTS In total, 7 UIAs were identified from the $305 \mathrm{MR}$ angiograms obtained. The prevalence of UIAs in first-degree relatives of patients with aSAH in the Hong Kong Chinese population was $2.30 \%$ (95\% $\mathrm{Cl} 1.02 \%-4.76 \%)$. This percentage was lower than the prevalence rate of $3.2 \%$ from a meta-analysis of the literature. The sizes of the UIAs detected ranged from $1.4 \mathrm{~mm}$ to $7.5 \mathrm{~mm} ; 85.7 \%$ of the UIAs detected in this study were $<5 \mathrm{~mm}$, in contrast to $66 \%$ noted in the literature. One of the UIAs identified underwent endovascular stent placement with a flow diverter. None of the UIAs identified ruptured or became symptomatic during a median follow-up period of 3.5 years.

CONCLUSIONS The prevalence of UIAs in first-degree relatives of patients with aSAH in the Hong Kong Chinese population was lower than that in Caucasians. At the same time, most of the UIAs detected in this study were small $(85.7 \%$ were $<5 \mathrm{~mm}$, vs $66 \%$ in a meta-analysis). With a similar incidence of aSAH in Hong Kong (7.5 per 100,000 person-years) as compared with data cited in the literature, the hypothesis that UIA rupture risk size threshold is different in Chinese patients should be further investigated.

http://thejns.org/doi/abs/10.3171/2015.4.JNS142938

KEY WORDS prevalence; unruptured intracranial aneurysm; Chinese; screening; Hong Kong; vascular disorders; subarachnoid hemorrhage; first-degree relative

$\mathrm{R}$ UPTURED intracranial aneurysms with subarachnoid hemorrhage (SAH) have high mortality and morbidity rates and affect relatively young people..$^{14} \mathrm{On}$ an individual level, aneurysmal SAH (aSAH) can cause a lot of anxiety within a family. ${ }^{11}$ On a public health level, it brings a considerable socioeconomic burden to society. ${ }^{12}$ With the increased accessibility of noninvasive imaging with higher resolution, earlier detection of unruptured intracranial aneurysms (UIAs) in asymptomatic individuals in the general population is more common. At the same time, the emergence of data on therapies such as endovascular coiling and pipeline stents provides more options with acceptable risks in the treatment of UIAs. It is not uncommon for family members to ask if they could possibly harbor an aneurysm as well, and whether they need screening. However, the role of screening has yet to be justified. Ninetyfive percent of the Hong Kong population is Chinese, and no previous epidemiological study has focused on the incidence of UIAs in Hong Kong Chinese ${ }^{19}$ These data could guide us in counseling family members with aSAH by of-

ABBREVIATIONS $\mathrm{ACOA}=$ anterior communicating artery; aSAH = aneurysmal $\mathrm{SAH} ; \mathrm{CTA}=\mathrm{CT}$ angiography; ICA = internal carotid artery; $\mathrm{MRA}=\mathrm{MR}$ angiography; $\mathrm{SAH}=$ subarachnoid hemorrhage; UIA = unruptured intracranial aneurysm; VA = vertebral artery.

SUBMITTED December 27, 2014. ACCEPTED April 7, 2015.

INCLUDE WHEN CITING Published online October 16, 2015; DOI: 10.3171/2015.4.JNS142938. 
fering screening or follow-up management. It could also have public health implications and guide future resource allocation and service development in Hong Kong. ${ }^{19}$

Therefore, our aim in this study was to generate data on the local prevalence of UIAs in first-degree relatives of patients with ruptured intracranial aneurysms and to explore the potential role of screening for first-degree relatives in our locality.

\section{Methods}

The study was approved by the Joint Chinese University of Hong Kong-New Territories East Cluster Clinical Research Ethics Committee and supported by a direct grant for research from the Chinese University of Hong Kong. Informed consent was obtained from participants and the study conformed to the Declaration of Helsinki. We identified first-degree relatives of consecutive patients with SAH from ruptured aneurysms admitted to a university hospital in Hong Kong (Prince of Wales Hospital) from June 2008 to December 2010.

Screening MRI was performed on a 3-T MRI machine (Achieva TX, Philips Medical Systems) using a standard head coil. Three-dimensional MR angiography (MRA) time-of-flight sequence of the whole brain was acquired using the following parameters: TR $23 \mathrm{msec}$, TE $3.5 \mathrm{msec}$, FOV $200 \times 200 \times 56 \mathrm{~mm}^{3}$, and resolution $0.4 \times 0.6 \times 0.7$ $\mathrm{mm}^{3}$. To complete the clinical scan, axial T1-weighted and T2-weighted, and coronal FLAIR sequences were additionally acquired in 5-mm-thick sections. In addition, confirmatory CT scans were performed on a 64-slice CT scanner (Lightspeed VCT, GE Healthcare). CT angiography (CTA) covering the region of the circle of Willis arteries was acquired in helical mode without tilting at 120 $\mathrm{kV}, 550 \mathrm{~mA}$, and $0.4-\mathrm{sec}$ rotation, and with the assistance of bolus tracking of intravenously injected contrast $(70 \mathrm{ml}$ Omnipaque 300, 3-3.5 ml/sec). Axial images were reconstructed at $0.625-\mathrm{mm}$ slices and used as source images.

MRA data were viewed as source images in multiplanar views, and in angiographic mode generated via maximal intensity projection. Scans were reviewed together by at least 2 neuroradiologists (D.Y.W.S., J.M.A., or T.C.Y.C.), and discrepancies were resolved by consensus. If MRA showed suspicious findings, CTA was performed to confirm the MRA findings.

In this study, MRA was used as the screening method of choice because it 1) is noninvasive compared with other imaging modalities, 2) does not use radiation, and 3) does not require intravenous contrast, while at the same time it maintains high sensitivity for aneurysm detection. ${ }^{8}$ The use of MRA also simulates the clinical setting whereby it is the most likely radiological investigation offered as a screening tool for asymptomatic individuals.

CTA was used for confirmatory imaging following our institutional practice of confirming incidental MRA findings in asymptomatic patients. CTA is less invasive and is not associated with a risk of stroke as compared with the gold standard of digital subtraction angiography; 6,16 both modalities are considered diagnostically equivalent. CTA is also more acceptable for asymptomatic patients with their natural inclination to not investigate further.
Moreover, the difference in measurements of aneurysm size between good quality 3D CTA and digital subtraction angiography is less then $1 \mathrm{~mm} .{ }^{20}$ Furthermore, 3D measurement of aneurysm size from CTA has high interobserver and intraobserver reliability. ${ }^{17}$

\section{Results}

There were 114 consecutive patients with $\mathrm{SAH}$ due to a ruptured aneurysm during the study period (Fig. 1). None of the screened patients had a first-degree relative with a history of aSAH. In total we identified 347 asymptomatic first-degree relatives. Three hundred five $(87.9 \%)$ consented to participate and were successfully recruited to undergo MRA. Forty-two asymptomatic first-degree relatives $(12.1 \%$ ) did not participate. The reasons these $12.1 \%$ did not undergo MRA included refusal to consent, claustrophobia, presence of nail in the eye, presence of metallic

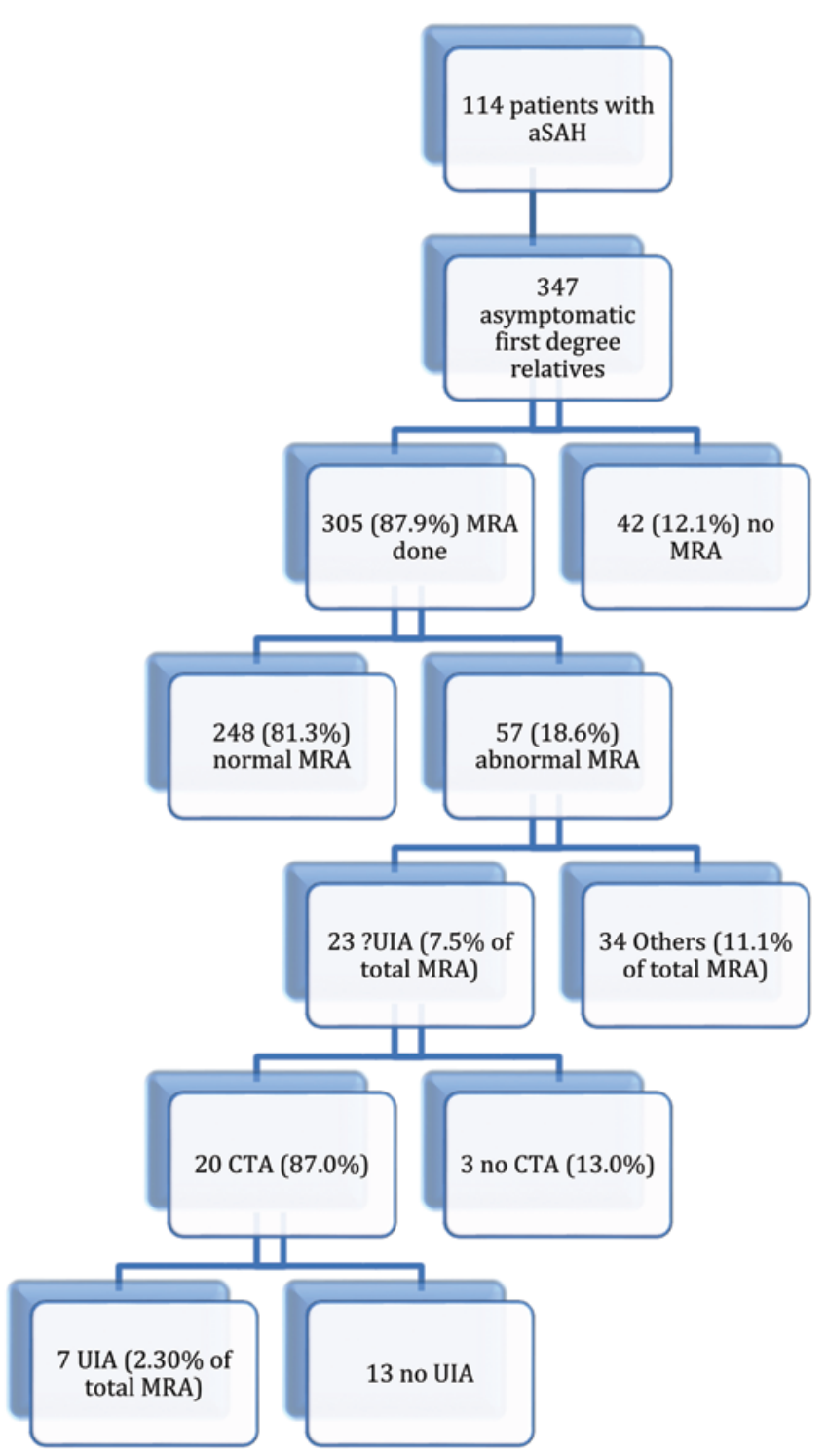

FIG. 1. Study participant flow diagram. Figure is available in color online only. 
screws and implant in body parts, physically not in Hong Kong, or failed to attend the MRA appointment.

Of $305 \mathrm{MR}$ angiograms performed, 248 (81.3\%) were normal, and 57 (18.6\%) were abnormal. Of the 57 abnormal MR angiograms, 23 (7.5\%) showed suspected UIAs, and $34(11.1 \%)$ had other findings including old infarction, vessel stenosis, small-vessel disease, old hemorrhage, and osteoma. Of the $23 \mathrm{MR}$ angiograms with suspected UIAs, 20 proceeded with CTA and 3 did not. Reasons for not undergoing CTA included refusal to consent, diabetes and taking metformin, and physically not in Hong Kong. Of the 20 with suspected UIAs that proceeded with CTA, 7 confirmed the presence of a UIA (Table 1) and 13 showed no abnormality (Table 2). The prevalence rate of UIAs in this population of first-degree relatives was thus $2.30 \%$ (95\% CI $1.02 \%-4.76 \%)$.

The sizes of the 7 UIAs detected ranged from 1.4 $\mathrm{mm}$ to $7.5 \mathrm{~mm}$ (Table 1). Six $(85.7 \%)$ of the 7 UIAs were smaller than $5 \mathrm{~mm}$ (range $1.4 \mathrm{~mm}-3.1 \mathrm{~mm}$ ). None of these 6 UIAs underwent an endovascular procedure or surgical intervention. The only UIA that was larger than $5 \mathrm{~mm}$ was a right intracranial vertebral artery (VA) saccular aneurysm in a 62-year-old woman. It measured $6.3 \mathrm{~mm}$ wide by $7.5 \mathrm{~mm}$ long. Stent placement using the Pipeline Embolization Device (ev3) was performed in September 2011. There had been no transient ischemic attack or stroke at the latest follow-up evaluation in October 2014. The latest CTA in October 2014 showed a patent right VA with no in-stent stenosis.

None of the UIAs identified ruptured or became symptomatic during the follow-up period, ranging from 31 to 55 months (median 43 months). Of the 3 suspected UIAs on MRA that did not undergo CTA, one was a $4.8 \times 2.5-\mathrm{mm}$ left internal carotid artery (ICA) aneurysm in a 46-yearold woman, one was a 2-mm left ICA aneurysm in a 44-year-old woman, and the third was a 2-mm anterior communicating artery (ACoA) aneurysm in a 48-year-old woman (Table 3).

To assess the impact of selection bias, for example, whether the prevalence could have been higher if the older population did not refuse MRA or CTA, we compared the age and sex ratios in the different groups. The ages of the 42 first-degree relatives who were identified and invited but did not undergo MRA ranged from 1 to 71 years old (mean 37.8 years old). After excluding those under the age of 21, the ages ranged from 21 to 71 years old (mean 47.8

TABLE 1. Computed tomography angiography findings in 7 patients with confirmed UIAs

\begin{tabular}{cl}
\hline Age (yrs), Sex & \multicolumn{1}{c}{ CTA Findings } \\
\hline $25, \mathrm{M}$ & 2-mm rt ICA aneurysm \\
\hline $43, \mathrm{M}$ & 1.4-mm It ICA aneurysm \\
\hline $49, \mathrm{~F}$ & $2.5-\mathrm{mm}$ It ICA aneurysm \\
\hline $57, \mathrm{~F}$ & 3.1-mm rt ICA aneurysm \\
\hline $58, \mathrm{M}$ & 2-mm rt ACA aneurysm \\
\hline $61, \mathrm{M}$ & 2-mm rt ICA aneurysm, 2.2-mm It ICA aneurysm \\
\hline $62, \mathrm{~F}$ & 7.5-mm rt VA aneurysm \\
\hline
\end{tabular}

$\mathrm{ACA}=$ anterior cerebral artery.
TABLE 2. Findings in 13 patients who underwent MRA for suspected UIAs with normal CTA results

\begin{tabular}{cl}
\hline Age (yrs), Sex & \multicolumn{1}{c}{ MRA Findings } \\
\hline $31, M$ & Lt PCoA aneurysm \\
\hline $39, \mathrm{M}$ & Rt MCA aneurysm \\
\hline $45, \mathrm{M}$ & Suspected ACoA aneurysm \\
\hline $47, \mathrm{M}$ & Suspected rt PCoA aneurysm \\
\hline $49, \mathrm{~F}$ & Suspected rt PCoA aneurysm \\
\hline $51, \mathrm{~F}$ & Bilat PCoA aneurysm \\
\hline $53, \mathrm{M}$ & Suspected rt ICA aneurysm \\
\hline $55, \mathrm{~F}$ & Aneurysm of the orbitofrontal branch of the It ACA \\
\hline $55, \mathrm{~F}$ & Suspected It PCoA aneurysm \\
\hline $60, \mathrm{~F}$ & Small aneurysm at origin of It PCoA \\
\hline $62, \mathrm{M}$ & Small aneurysm in the rt ICA \\
\hline $62, \mathrm{~F}$ & Small aneurysm in the It ICA at the ophthalmic artery \\
\hline $64, \mathrm{M}$ & Suspected It PCoA aneurysm
\end{tabular}

$\mathrm{MCA}=$ middle cerebral artery; $\mathrm{PCOA}=$ posterior communicating artery.

years old). There were 24 males and 18 females (ratio: 1 to 0.75 ). The ages of the patients with 7 confirmed UIAs ranged from 25 to 62 years old (mean age 50.7 years old). There were 4 men and 3 women (ratio: 1 to 0.75 ). The ages of the 13 patients with suspected UIAs on MRA but normal CTA results ranged from 31 to 64 years old (mean 51.8 years old). There were 7 men and 6 women (ratio: 1 to 0.86 ).

\section{Discussion}

In this study, the prevalence of UIAs in asymptomatic Hong Kong Chinese with 1 first-degree relative with a positive history of aSAH was $2.30 \%(95 \%$ CI $1.02 \%-$ $4.76 \%$ ), which is lower than rates cited in the literature. A systematic review and meta-analysis of prevalence studies showed that the prevalence of UIA was $3.2 \%$ (95\% CI $1.9 \%-5.2 \%$ ) in a population without comorbidity. A prevalence ratio of 3.4 (95\% CI 1.9-5.9) was found for a positive family history of intracranial aneurysm or SAH. ${ }^{13}$ One screening study showed that $4 \%$ (95\% CI $2.6 \%-5.8 \%$ ) of patients with aSAH had first-degree relatives with UIA. ${ }^{10}$

At the same time, the incidence of aSAH in Hong Kong has been reported to be 7.5 (range 6.9-8.2) per 100,000 person-years. ${ }^{18}$ This is comparable to the data cited in the literature, in which the incidence of aSAH has been estimated to be approximately 7.8 (95\% CI 7.2-8.4) per 100,000 person-years in most populations. ${ }^{9,12,13}$ With the discrepancy in prevalence of UIAs, yet a similar incidence of aSAH, one probable explanation is that UIAs in Hong

TABLE 3. Magnetic resonance angiography findings in 3 patients with suspected UIAs who did not undergo CTA

\begin{tabular}{cl}
\hline Age (yrs), Sex & \multicolumn{1}{c}{ MRA Findings } \\
\hline $44, \mathrm{~F}$ & 2-mm It ICA aneurysm \\
\hline $46, \mathrm{~F}$ & 4 -8-mm It ICA aneurysm \\
\hline $48, \mathrm{~F}$ & 2-mm ACoA aneurysm \\
\hline
\end{tabular}


Kong Chinese ruptured at a smaller size. This coincides with our data that showed most of the UIAs identified were $<5 \mathrm{~mm}$.

Most of the UIAs detected in our study were small, in which 6 of 7 were $<5 \mathrm{~mm}(85.7 \%)$. With assumptive analysis of the 3 participants with suspected UIAs on MRA who did not undergo CTA, 2 more may also have harbored a true UIA (both ICA aneurysms, which were $2 \mathrm{~mm}$ and $4.8 \mathrm{~mm}$ in size, respectively); therefore $8(88.9 \%)$ of these 9 were $<5 \mathrm{~mm}$. Both of these values were higher than the value of $66 \%$ from the meta-analysis. ${ }^{14}$ A previous study from our center had suggested that ruptured intracranial aneurysms are smaller in Asians. ${ }^{18}$ With a similar incidence of aSAH in Hong Kong (7.5 per 100,000 personyears) as compared with the data from the literature, the hypothesis that UIA rupture risk size threshold is different in Chinese should be further investigated.

Screening with either CTA or MRA is usually recommended for first-degree relatives of families with 2 or more members with aneurysms or aSAH. ${ }^{5}$ Moreover, a long-term serial screening study for UIAs in families with 2 or more aSAHs or UIAs showed a substantial yield of $3 \%-5 \%$ of newly identified UIAs after more than 10 years of follow-up and 2 initial negative screenings, ${ }^{3}$ supporting the rationale of offering screening in this population.

Currently, screening is not generally recommended if there is only 1 family member with an aneurysm or aSAH. ${ }^{1}$ However, the latest data have suggested a strategy of screening once for UIA in persons with 1 first-degree family member with aSAH during that person's lifetime; screening at an age between 30 and 55 years was the most cost-effective with the lowest costs per quality-adjusted life years. ${ }^{2}$

At the same time, proper counseling is crucial in the process of offering screening. This is particularly important if the UIAs identified are small and do not undergo intervention, because the potential benefits of a positive result of screening could be offset by depression and a reduction in quality of life. ${ }^{15}$ This is consistent with the fact that none of the small UIAs $(<5 \mathrm{~mm})$ identified in our study underwent intervention. This is justifiable because this strategy is balanced against the potential risks of intervention, be they endovascular coiling or clip placement. ${ }^{4}$

There were limitations in the current study. The number of first-degree relatives with UIAs was small. However, as the prevalence was approximately $2 \%$, the overall UIAs yielded could have been increased by approximately 2 after screening of about another 100 asymptomatic persons using MRA, which was limited by funding and resources for our study. Of the eligible first-degree relatives, $12.1 \%$ did not undergo MRA for various reasons. No interobserver reliability was assessed. MRA was performed at the University Radiology Department's neuroradiology imaging session. The angiograms were read and reported by the consensus of 2 or 3 neuroradiologists, as is our usual standard of practice. The aim of the study was to generate data on the prevalence of UIAs, and interobserver reliability was thus not incorporated into the study design. The diagnostic yield might be different with a different MRI machine and protocol, which was not addressed in the current study. Three first-degree relatives with MRAdetected UIAs did not undergo CTA. This group could have harbored UIAs. However, it was limited by subjects' refusal to consent, which in turn was an objective reflection of the practical difficulty in this clinical situation. There was no family with 2 or more first-degree relatives with a ruptured aneurysm and SAH during the study period. Thus, we cannot comment as to whether there was any yield in families with 2 or more first-degree relatives in our study. There was no family with more than 1 firstdegree relative who screened positive for an unruptured aneurysm. Hence we are unable to investigate the rate of UIAs in those with a single affected family member versus those with more than 1 member.

Because of not only the relative low incidence of UIAs but also the fact that most of the aneurysms found were very small and probably do not need to be treated, we do not recommend the practice of general screening of firstdegree relatives of patients with aSAH if there is no history of a second immediate member of the family having had an aneurysm. At the same time, we need to acknowledge the existing uncertainty on the natural history of UIAs.?

\section{Conclusions}

The prevalence of UIAs in first-degree relatives of patients with aSAH in Hong Kong Chinese in this study was $2.30 \%$ (95\% CI $1.02 \%-4.76 \%$ ), which is lower than that in Caucasians, while most of the UIAs detected in our study were small $(85.7 \%$ were $<5 \mathrm{~mm}$, vs $66 \%$ in a meta-analysis).

\section{Acknowledgment}

This study was supported by a direct grant for research from the Chinese University of Hong Kong (no. MD09731).

\section{References}

1. Bederson JB, Awad IA, Wiebers DO, Piepgras D, Haley EC $\mathrm{Jr}$, Brott T, et al: Recommendations for the management of patients with unruptured intracranial aneurysms: A Statement for healthcare professionals from the Stroke Council of the American Heart Association. Stroke 31:2742-2750, 2000

2. Bor AS, Koffijberg H, Wermer MJ, Rinkel GJ: Optimal screening strategy for familial intracranial aneurysms: a cost-effectiveness analysis. Neurology 74:1671-1679, 2010

3. Bor AS, Rinkel GJ, van Norden J, Wermer MJ: Long-term, serial screening for intracranial aneurysms in individuals with a family history of aneurysmal subarachnoid haemorrhage: a cohort study. Lancet Neurol 13:385-392, 2014

4. Brainin M: Finding silent cerebral aneurysms: the importance of doing nothing. J Neurol Neurosurg Psychiatry 77:713, 2006

5. Brown RD Jr, Broderick JP: Unruptured intracranial aneurysms: epidemiology, natural history, management options, and familial screening. Lancet Neurol 13:393-404, 2014

6. Dion JE, Gates PC, Fox AJ, Barnett HJ, Blom RJ: Clinical events following neuroangiography: a prospective study. Stroke 18:997-1004, 1987

7. Etminan N, Beseoglu K, Barrow DL, Bederson J, Brown RD $\mathrm{Jr}$, Connolly ES Jr, et al: Multidisciplinary consensus on assessment of unruptured intracranial aneurysms: proposal of an international research group. Stroke 45:1523-1530, 2014 
8. Li MH, Li YD, Tan HQ, Gu BX, Chen YC, Wang W, et al: Contrast-free MRA at 3.0 T for the detection of intracranial aneurysms. Neurology 77:667-676, 2011

9. Linn FH, Rinkel GJ, Algra A, van Gijn J: Incidence of subarachnoid hemorrhage: role of region, year, and rate of computed tomography: a meta-analysis. Stroke 27:625-629, 1996

10. Magnetic Resonance Angiography in Relatives of Patients with Subarachnoid Hemorrhage Study Group: Risks and benefits of screening for intracranial aneurysms in first-degree relatives of patients with sporadic subarachnoid hemorrhage. N Engl J Med 341:1344-1350, 1999

11. Molyneux AJ: Is screening of relatives for cerebral aneurysms justified? Lancet Neurol 13:343-344, 2014

12. Rivero-Arias O, Gray A, Wolstenholme J: Burden of disease and costs of aneurysmal subarachnoid haemorrhage (aSAH) in the United Kingdom. Cost Eff Resour Alloc 8:6, 2010

13. van Gijn J, Kerr RS, Rinkel GJE: Subarachnoid haemorrhage. Lancet 369:306-318, 2007

14. Vlak MH, Algra A, Brandenburg R, Rinkel GJ: Prevalence of unruptured intracranial aneurysms, with emphasis on sex, age, comorbidity, country, and time period: a systematic review and meta-analysis. Lancet Neurol 10:626-636, 2011

15. Wermer MJH, van der Schaaf IC, Van Nunen P, Bossuyt PMM, Anderson CS, Rinkel GJE: Psychosocial impact of screening for intracranial aneurysms in relatives with familial subarachnoid hemorrhage. Stroke 36:836-840, 2005

16. Willinsky RA, Taylor SM, terBrugge K, Farb RI, Tomlinson G, Montanera W: Neurologic complications of cerebral angiography: prospective analysis of 2,899 procedures and review of the literature. Radiology 227:522-528, 2003

17. Wong GKC, Leung JHY, Yu JWL, Woo PYM, Tan HB, Siu DYW, et al: Reliability of computed tomographic angiography 3-D method of ruptured intracranial aneurysm measurements. Surg Pract 17:97-100, 2013

18. Wong GKC, Teoh J, Chan EKY, Ng SCP, Poon WS: Intracranial aneurysm size responsible for spontaneous subarachnoid haemorrhage. Br J Neurosurg 27:34-39, 2013

19. Wong GKC, Wun Tam YY, Zhu XL, Poon WS: Incidence and mortality of spontaneous subarachnoid hemorrhage in Hong Kong from 2002 to 2010: a Hong Kong hospital authority clinical management system database analysis. World Neurosurg 81:552-556, 2014

20. Wong GKC, Yu SCH, Poon WS: Radiological measurements of dimensions of acutely ruptured internal carotid artery aneurysm: a comparative study between computed tomographic angiography and digital subtraction angiography. Clin Pract 2:e75, 2012

\section{Disclosure}

The authors report no conflict of interest concerning the materials or methods used in this study or the findings specified in this paper.

\section{Author Contributions}

Conception and design: Wong, Ahuja. Acquisition of data: Wong, Abrigo, Cheung, Siu, Ahuja. Analysis and interpretation of data: Wong, Chan, Siu. Drafting the article: Wong, Chan. Critically revising the article: all authors. Reviewed submitted version of manuscript: all authors. Approved the final version of the manuscript on behalf of all authors: Wong. Statistical analysis: Wong, Chan. Administrative/technical/material support: all authors. Study supervision: Wong.

\section{Supplemental Information}

\section{Previous Presentation}

Part of the paper was previously presented as an oral presentation on November 2, 2013, at the Conjoined Congress of the 5th World Conference of Intracerebral Hemorrhage (ICH) \& 20th Annual Scientific Meeting of the Hong Kong Neurosurgical Society, in Hong Kong.

\section{Correspondence}

George Kwok Chu Wong, 4/F Department of Surgery, Prince of Wales Hospital, 30-32 Ngan Shing St., Shatin, NT 852, Hong Kong. email: georgewong@surgery.cuhk.edu.hk. 